\title{
Consolidation of Host-Based Mobility Management Protocols with Wireless Mesh Network
}

\author{
Wei Siang Hoh ${ }^{1}$, Bi-Lynn Ong ${ }^{2}$, R Badlishah Ahmad ${ }^{3}$, Hasnah Ahmad ${ }^{4}$ \\ \{weisiangkelvin1990@gmail.com ${ }^{1}$, drlynn@unimap.edu.my ${ }^{2}$, badli@ unimap.edu.my $^{3}$ \} \\ Universiti Malaysia Perlis (UniMAP), School of Computer and Communication Engineering, \\ 02600, Arau, Perlis, Malaysia ${ }^{1,2,3}$
}

\begin{abstract}
The number of mobile devices increases exponentially and it becomes the trends and needs of human. With the increasing demands for new data and real-time services, there is a need of wireless Internet networks that can support different traffic characteristics and different Quality of Service (QoS) guarantees. All these Internet network mobile device services are supported by the mobility management protocols and its affects the QoS of the Internet. Having known the importance of mobility management protocol, we consolidate MIPv6, HMIPv6, FMIPv6 and FHMIPv6 with Wireless Mesh Network (WMN) into one environment. We identify, analyze, and compare the performance of Host-Based mobility management protocols integrate with WMN in terms of latency, throughput and packet loss ratio. At the end of this research, it is proven that the design and development of FHMIPv6 with WMN performs better as compared to the others Mobile Internet Protocols over the Internet using NS-2 Network Simulation software.
\end{abstract}

Keywords: Host-Based Mobility Management Protocols, MIPv6, HMIPv6, FMIPv6, FHMIPv6, Wireless Mesh Network (WMN)

\section{Introduction}

The Internet consolidated itself as a very powerful platform that has forever changed the way human communicates and their behavior. The mobile communications technology had made it possible for much greater reach of the Internet and increase the number of Internet users through the mobile devices in wireless environment. These cause the connection distortion, network congestion and disconnection. The reason is because of the huge number of Internet users. Therefore, mobility management protocols are introduced to solve the connection distortion, network congestion and disconnection issues. The mobility management is the essential part for mobile devices that are automatically connect to Internet while simultaneously can roam freely without disturbing the communication. Mobility management provides routing support and permits IP nodes using either IPv4 or IPv6 to seamlessly roam among IP subnetworks and media types. This is also called as mobility support protocols. Host-Based mobility management protocols are one of the parts of mobility management protocols. It includes Mobile Internet Protocol version 6 (MIPv6) [1] and its enhancement such as Fast Handover Mobile Internet Protocol version 6 (FMIPv6) [2], Hierarchical Mobile Internet Protocol version 6 (HMIPv6) [3] and Fast Handover for Hierarchical Mobile Internet Protocol version 6 (FHMIPv6). 
WMN can be connected to wireless networks such as worldwide interoperable microwave access (WiMAX), generic wireless fidelity (Wi-Fi); cellular and sensor networks. Third (3G) and Fourth (4G) Generation networks include all Internet Protocol (IP) which are wired and wireless networks interworks together as heterogeneous networks [4]. However, the challenge is to connect to Host-Based mobility management. Host-Based Mobility Management protocols rely on the good performance of an infrastructure-based network. However, a typical WMN topology tends to be an unplanned graphs and routes of it dynamically changes [5]. Mobility management provides an undisrupted support of real-time and non-real-time services to mobile network users. Additionally, mobility management also facilitates the maintenance of connections for users on the move when they change their points of attachment from one access point (AP) to another. Host-Based mobility allows a Mobile Node (MN) to change its point of attachment to the network, without interrupting IP packet delivery to or from the node. The current location of all the MNs in the network is maintained by Access Network Procedures.

In this research paper, all the Host-Based Mobility Management Protocols are investigated firmly on Wireless Mesh Network topology environment. MIPv6, HMIPv6, FMIPv6 and FHMIPv6 are developed and analyzed in Wireless Mesh Network (WMN) environment which are considering the performance parameters: packet delivery ratio, delay/latency and throughput. The Wireless Mesh Network (WMN) topology are developed by using network simulation software and the result obtained are analyzed to agree the best mobility management protocols in different Wireless Mesh Network topology scenarios.

\section{Related Works}

Jong-Hyouk Lee, et. al [6] had investigated "Comparative Handover Performance Analysis of IPv6 Mobility Management Protocols". The researchers compared the Host-Based mobility management protocols and Network-Based mobility management protocols to identify the optimized routing protocol for mobile network. The Host-Based mobility management protocols include Mobile IPv6 and its extensions such as Fast Mobile IPv6 and Hierarchical Mobile IPv6 while Network- Based mobility management protocols include Proxy Mobile IPv6 (PMIPv6) and Fast Proxy Mobile IPv6 (FPMIPv6). These mobility management protocols have been standardized. The existing IPv6 mobility management protocols are developed by the IETF and have been analyzed and compared in terms of handover latency, handover blocking probability, and packet loss. The conducted analysis results can be used to identify each mobility management protocol's characteristic and performance indicators. The results obtained are used to facilitate decision making in development a new mobility management protocol.

K Vasu, et. al [7], had investigated "MIPv6 Protocols: A Survey And Comparative Analysis". The researchers had performed various mobility management protocols in terms of handover latency and the number of hops is needed to evaluate these protocols. The IPv6 mobility management protocols such as MIPv6, FMIPv6 (Reactive), FMIPv6 (Predictive), HMIPv6, PMIPv6, FPMIPv6 (Reactive), and FPMIPv6 (Predictive) are analyzed and compared in terms of average hop delay, wireless link delay, wired part delay, binding update and registration delay. PMIPv6 and FPMIPv6 have been compared with the Host-Based mobility management protocols to make a decision that suits the future networks. The conclusion that the authors made among these protocols : reactive mode protocols performs 
better in terms of delay compare to predictive based protocols. The performance are measured in terms of delay during AP to MAG/AR, and binding update/registration components. Whereas predictive based protocols performs better performance in term wireless link delay for faster radio access technologies and performs rather slower performance for slower radio access technologies.

J. H Sun, et. al [8], had investigated "Mobility Management Techniques for Next Generation Wireless Networks". The researcher had performed macro and micro mobility protocols in terms of handover performance. The macro and micro mobility protocols such as Mobile Internet Protocol version 6 (MIPv6), Fast Handover Mobile Internet Protocol version 6 (FMIPv6), Hierarchical Mobile Internet Protocol version 6 (HMIPv6) and Fast Handover for Hierarchical Internet Protocol version 6 (FHMIPv6) and Proxy Mobile Internet Protocol version 6 (PMIPv6). These protocols are analyzed and compared in term of handover latency. The conclusion that the authors made that the best handover latency is achieved by FHMIPv6. The result is signalling load reduction, improvement in latency and less packet losses apart from aiding the handover process.

\section{Terminology}

\subsection{Host-Based Mobility Management Protocols}

Host-Based mobility management protocols include Mobile Internet Protocol version 6 (MIPv6), and its enhancement such as Fast Handover Mobile Internet Protocol version 6 (FMIPv6), Hierarchical Mobile Internet Protocol version 6 (HMIPv6) and Fast Handover for Hierarchical Mobile Internet Protocol version 6 (FHMIPv6). Host-Based mobility management protocols are deployable in wireless mobile communication infrastructures, communication service providers and standards development organizations [9]. These mobility management protocols have identified that such conventional solutions for mobility service are not suitable; in particular, for telecommunication service. The reason is because the mobile node $(\mathrm{MN})$ is required to perform mobility functionalities at its network protocol stack inside, and thus, modifications or upgrades of the $\mathrm{MN}$ are needed. It obviously increases the operation expenses and complexity for the MN. Hence, the extension of Mobile IPv6 (MIPv6) had been introduced to overcome the handover latency problem. The extension of MIPv6 includes HMIPv6, FMIPv6 and FHMIPv6.

\section{Mobile Internet Protocol version 6 (MIPv6)}

Internet Engineering Task Force (IETF) brought into use of Mobile Internet Protocol version 6 (MIPv6) to allow mobile nodes $(\mathrm{MN})$ to be reachable and maintained on-going connection while changing location within topology without changing the allocated IP address. The operation begins as MN detects movement to a Foreign Agent (FA) and autoconfigures itself with a New Care of Address (NCoA) using either stateful or stateless method. MN sends Binding Update (BU) to its Home Agent (HA) to notify the new address and HA returns back Binding Acknowledgment (BAck). Then, all packets are tunneled to MN's NCoA with the help of HA as HA encapsulates packets and sends to MN's NCoA and MN decapsulates the packets received from HA. An additional mode for MIPv6 is Route Optimization (RO). It allows the packets to be delivered using shortest path. This process 
requires $\mathrm{MN}$ to register its current Binding to Corresponding Node $(\mathrm{CN})$. This allows $\mathrm{CN}$ to triangulate packets to be delivered to $\mathrm{MN}$ without concerning HA. This measure reduces congestion at MN's HA and Home Link.

\section{Hierarchical Mobile Internet Protocol version 6 (HMIPv6)}

The Internet Engineering Task Force (IETF) has introduced the HMIPv6 based on its predecessor MIPv6 and has implemented new technologies to it to ensure the increment in the performance of mobile networking. One of the latest features in HMIPv6 is Mobility Anchor Point (MAP). The introduction of MAP in HMIPv6 has improved the handover latency and reduced the amount of signaling between the Mobile Node (MN), its Correspondent Nodes (CNs), and its Home Agent (HA) [10]. Thus, HMIPv6 is more efficient compared to the previous MIPv6 in terms of handover and broadcasting. The technology also improves the inter network connection and smoothen the users' intermittent connections.

\section{Fast Handover Mobile Internet Protocol version 6 (FMIPv6)}

FMIPv6 is another initiative by the Internet Engineering Task Force (IETF) to improve the mobile network for the mobile users. The FMIPv6 is also designed based on the previous version of MIPv6. The handover latency is defined as a duration from reception of last packet via the previous link to reception of the first packet via new link. This handover latency results from standard Mobile IPv6 procedures, namely movement detection, new Care of Address $(\mathrm{CoA})$ configuration and confirmation, and Binding Update (BU) as well as link switching delay and these procedures are time consuming tasks. High handover latency is unacceptable to real-time traffic such as VoIP. To reduce the handover latency, FMIPv6 is introduced and it supports a fast handover procedure allowing starting handover in advance a movement.

\section{Fast Handover for Hierarchical Mobile Internet Protocol version 6 (FHMIPv6)}

FHMIPv6 is the combination of two mechanisms that are Fast Handover Mobile Internet Protocol version 6 (FMIPv6) and Hierarchical Mobile Internet Protocol version 6 (HMIPv6). Fast Handover for Hierarchical Mobile IPv6 (FHMIPv6) reduces the signaling overhead and Binding Update (BU) delay during handover by using HMIPv6 procedures. Furthermore, movement detection latency and new CoA configuration delay during handover are reduced by utilizing FMIPv6 processes.

The FHMPv6 contains the Router Solicitation for Proxy Advertisement (RtSolPr), Proxy Router Advertisement (PrRtAdv) and Fast Binding Update (FBU) technology from the FMIPv6 mechanism and also the Mobility Anchor Point (MAP) technology from the HMIPv6 mechanism combined into one single technology FHMIPv6. When the MN associates with a new MAP domain, HMIPv6 procedures are performed with the HA and the Mobility Anchor Point (MAP). If the MN moves from a previous AR (pAR) to a new AR (nAR) within the domain, it follows the local BU process of HMIPv6. Packets sent to the MN by the CN during handover are tunneled by the MAP enroute for the nAR.

\subsection{Wireless Mesh Network}

A network of wireless mesh consists of mesh clients, mesh routers and gateways. Mesh clients are such as cell phones, laptops and other wireless devices. Mesh routers forward 
traffic from and to gateways. The gateway may or need not to connect to Internet. Sometimes a single network of radio nodes working in a coverage area is called a mesh cloud. A mesh network is reliable and offers redundancy. With a powerful multiservice mesh platform, organizations can combine formerly separate voice, video and data networks onto a single network. The converged network is simpler to manage and operate, while the organization retains control over the delivery of multiple services. In addition, less devices are required. Hence, the network construction cost are low. A mesh is resilient and low maintenance. A modern mesh network automatically discovers the best route through the network and operates smoothly even if a mesh link broken down or a node fails. This is due to the network that is self-forming and self-healing. Besides that, the administration and maintenance costs for WMN are lower. In addition, a wireless mesh overcomes the line-of-sight issues that may occur when a space is crowded with buildings or industrial equipment.

\section{Simulation Design}

To perform a comparison between MIPv6, HMIPv6, FMIPv6 and FHMIPv6 mobility protocols, some configurations and parameters need to be fixed to obtain the optimum results for each mobility management protocol. The environment for all Host-Based mobility management protocols are set up in Wireless Mesh Network topology environment and the data rate is fixed in $10 \mathrm{Mbps}$. Table I below shows the type of parameters and values that are needed to fix for the whole simulation process.

Table 1. Type of Parameters and Value

\begin{tabular}{|l|c|}
\hline Wireless Mesh Network Data Rate & $10 \mathrm{Mbps}$ \\
\hline Window Size (byte) & 32 \\
\hline Transport Protocol & TCP \\
\hline Link Delay & $2 \mathrm{~ms}$ \\
\hline
\end{tabular}

The network topology consists of MIPv6, HMIPv6, FMIPv6, FHMIPv6 and WMN. Inter network section comprises of 8 routers with 5 wired routers and 3 wireless routers that are accomplished as base stations. Intra network portion includes 9 Wireless Mesh routers which has been setup in a grid formation to maximize the coverage area. Figure 1 shown the simulation design of inter and intra networks environment for Host-Based mobility management protocol. Table 2 below shows configuration details for inter and intra networks topology of each link and node.

Table 2. Configuration Details for Inter and Intra Networks Topology

\begin{tabular}{|c|c|c|}
\hline Link Connection & Link Speed & Queue Type \\
\hline CN - N1 & $100 \mathrm{Mbps}$ & RED \\
\hline HA - N1 & $100 \mathrm{Mbps}$ & RED \\
\hline N1 - MAP & $100 \mathrm{Mbps}$ & RED \\
\hline MAP - N2 & $10 \mathrm{Mbps}$ & RED \\
\hline MAP - N3 & $10 \mathrm{Mbps}$ & RED \\
\hline N2 - pAR & $1 \mathrm{Mbps}$ & DropTail \\
\hline N3 - nAR & $1 \mathrm{Mbps}$ & DropTail \\
\hline
\end{tabular}




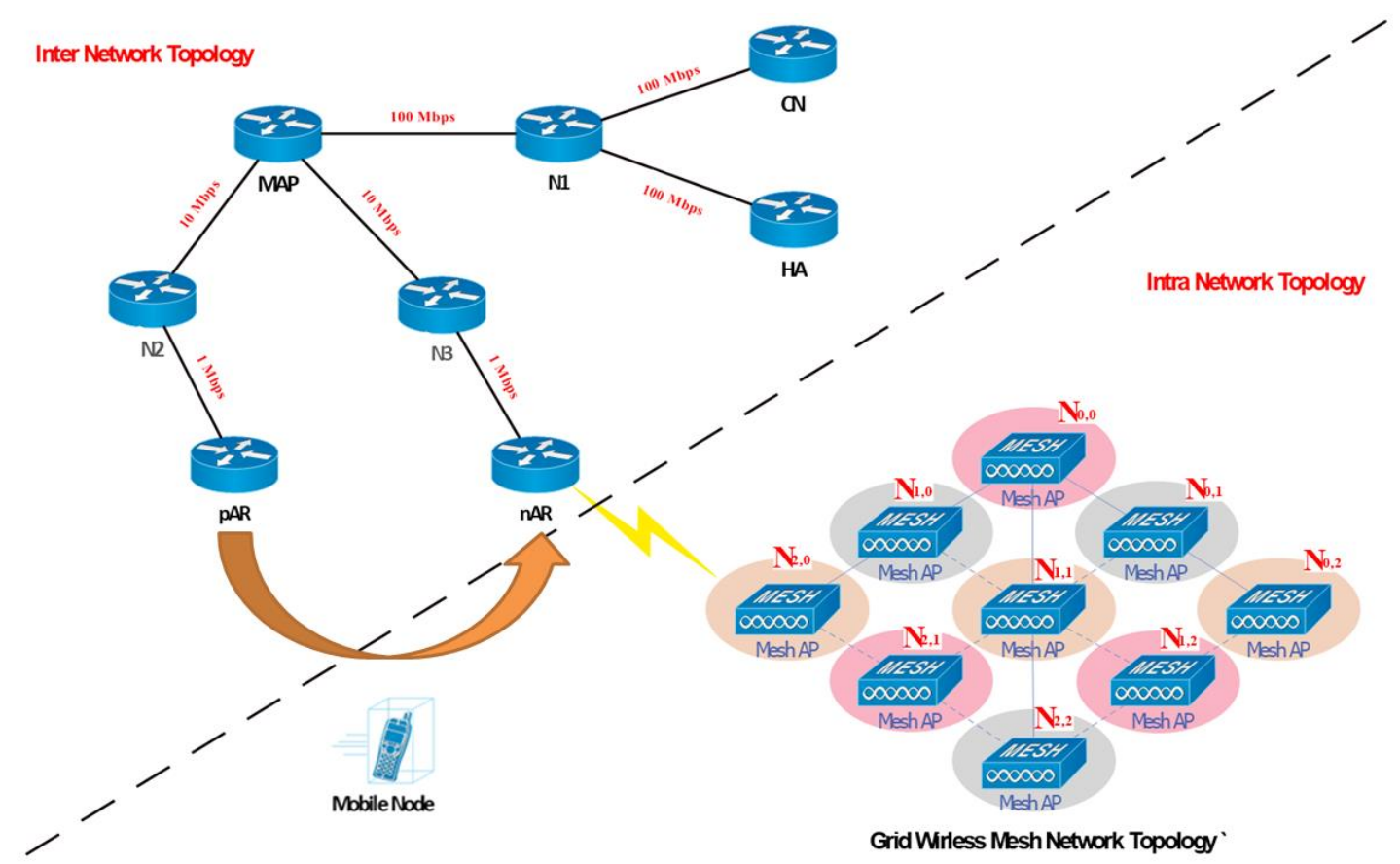

Fig. 1. Inter and Intra Networks Environment

\section{Results and Discussion}

Simulation results are presented in this section. In Table 3, the result of each performance metric for all Host-Based mobility management protocols in Wireless Mesh Network (WMN) environment are shown. The packet sizes used for simulation are started from 256 bytes, and increase to 512 bytes, 1024 bytes, and 2048 bytes. The Host-Based mobility management protocols include MIPv6, HMIPv6, FMIPv6 and FHMIPv6.

Table 3. Performance of Various Types of Host-Based Mobility Management Protocols in WMN Environment 


\begin{tabular}{|c|c|c|c|}
\hline \multicolumn{4}{|c|}{ Mobile Internet Protocols version $6(\mathrm{MIPv} 6)$ with WMN } \\
\hline Packet Size (bytes) & Latency Mean (ms) & Packet Delivery Ratio (\%) & Thoughput (kbps) \\
\hline 256 & 175 & 74.68 & 69617.23 \\
\hline 512 & 179 & 75.22 & 70860.98 \\
\hline 1024 & 178 & 74.66 & 70215.13 \\
\hline 2048 & 183 & 82.43 & 91023.06 \\
\hline \multicolumn{4}{|c|}{ Hierarchical Mobile Internet Protocol version 6 (HMIPv6) with WMN } \\
\hline Packet Size (bytes) & Latency Mean (ms) & Packet Delivery Ratio (\%) & Thoughput (kbps) \\
\hline 256 & 201 & $\mathbf{8 1 . 7 6}$ & 77824 \\
\hline 512 & 204 & 82.22 & 78807.04 \\
\hline 1024 & 202 & 84.51 & 80858.21 \\
\hline 2048 & 237 & 92.65 & 104133.43 \\
\hline \multicolumn{4}{|c|}{ Fast Handover Mobile Internet Protocol version 6 (FMIPv6) with WMN } \\
\hline Packet Size (bytes) & Latency Mean (ms) & Packet Delivery Ratio (\%) & Thoughput (kbps) \\
\hline 256 & 195 & 79.87 & 75243.53 \\
\hline 512 & 200 & 81.05 & 77672.9 \\
\hline 1024 & 198 & 81.51 & 77987.84 \\
\hline 2048 & 215 & 89.65 & 100761.6 \\
\hline \multicolumn{4}{|c|}{ Fast Handover for Hierarchical Mobile Internet Protocol version 6 (FHMIPv6) with WMN } \\
\hline Packet Size (bytes) & Latency Mean (ms) & Packet Delivery Ratio (\%) & Thoughput (kbps) \\
\hline 256 & 200 & 81.76 & 77824 \\
\hline 512 & 202 & 82.22 & 78807.04 \\
\hline 1024 & 201 & 84.51 & 80858.21 \\
\hline 2048 & 234 & 92.65 & 104133.43 \\
\hline
\end{tabular}

Based on the results obtained from the simulation experiments, MIPv6 with WMN performs well in term of latency mean. The reason that MIPv6 with WMN has low latency is because the packets have been dropped that contribute to a low Packet Delivery Ratio and Throughput. The highest Packet Delivery Ratio that can be reached is only $82.43 \%$ and in the same time the Throughput only reaches 91023.06 kbps. HMIPv6 and FHMIPv6 with WMN perform well in term of Throughput and Packet Delivery Ratio (PDR). This is because the Mobile Anchor Point (MAP) is implemented and the hierarchical movement pattern performs better. The FHMIPv6 with WMN performs slightly better that HMIPv6 in term of latency mean. FMIPv6 with WMN has a fair performance outcome as it is based on the anticipated handover with a slightly lower Packet Delivery Ratio and Throughput compare to HMIPv6 and FHMIPv6. 


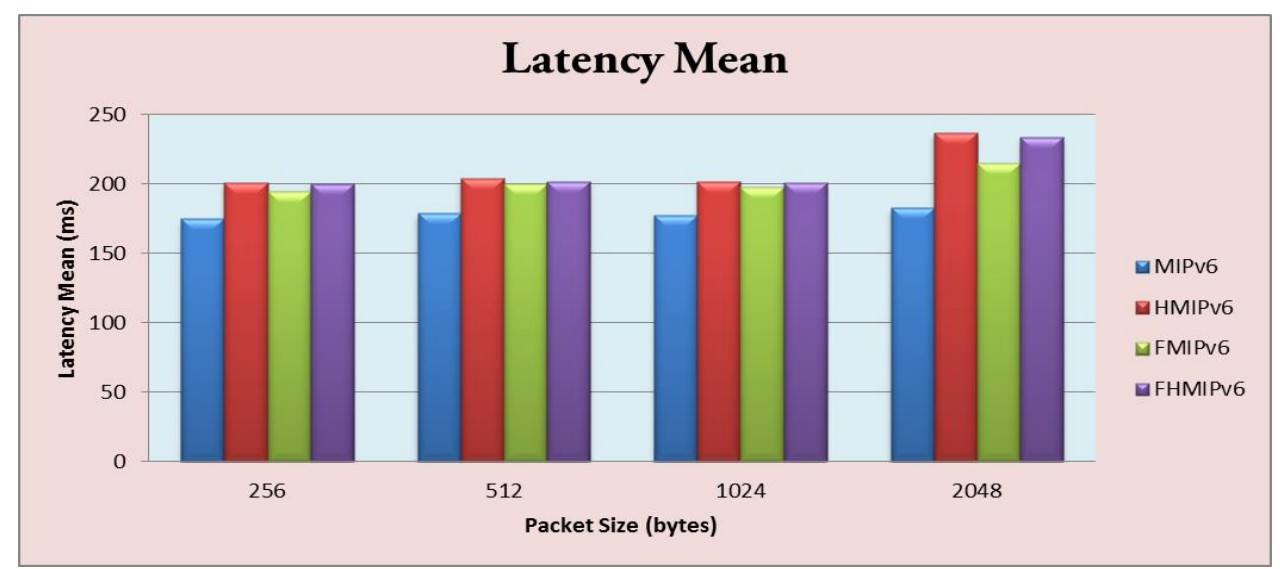

Fig. 2. Latency Mean Chart

Having studied Figure 2 above, it is observed that as the packet size increases, latency mean is increased. The reason is because as the packet size increases, the network needs more time to send packets over the Mobile Internet and through Wireless Mesh Network. The handoff latency is also included. However, in Wireless Mesh Network, it has minimized the time for handover process. Additionally, it is observed that MIPv6 performs better compared to HMIPv6, FMIPv6 and FHMIPv6. It is followed by FMIPv6 that performs better than HMIPv6 and FHMIPv6. Although HMIPv6 and FHMIPv6 have been implemented with MAP, but FHMIPv6 has slightly lower latency mean compared with HMIPv6. However, latency mean for Host-Based mobility management protocols do not have many differences. Thus, it can be concluded that all Host-Based mobility management protocols have not much improvement between the mechanisms with the consolidation with WMN.

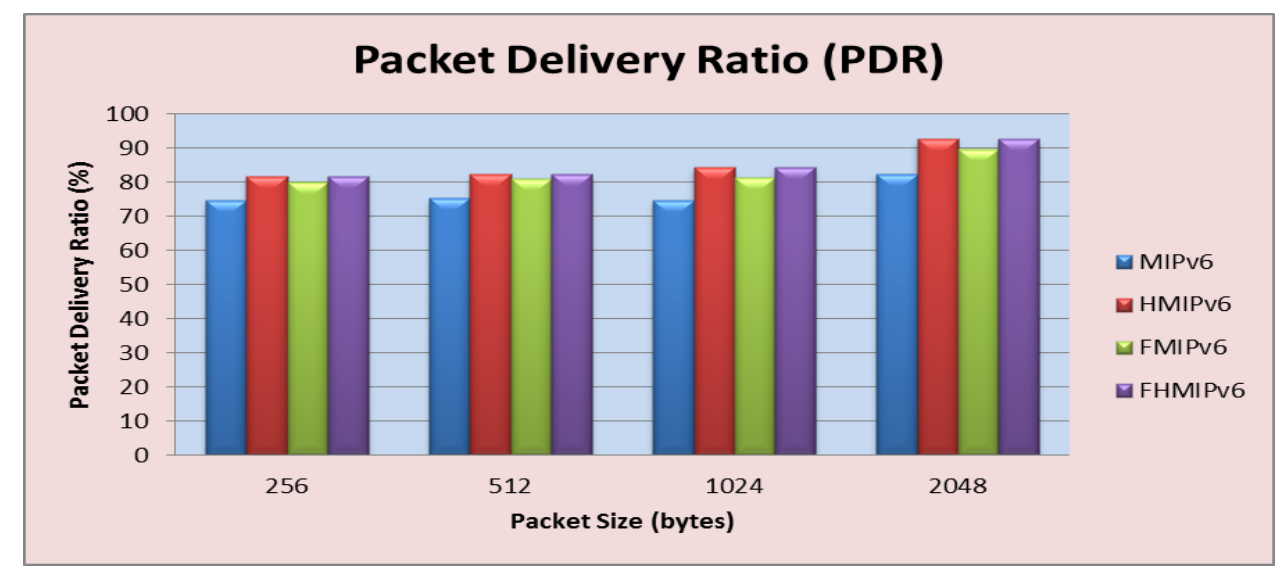

Fig. 3. Packet Delivery Ratio (PDR) Chart

As shown in Figure 3, the packet delivery ratio of MIPv6, HMIPv6, FMIPv6 and FHMIpv6 for various packet sizes are presented. It can be observed that HMIPv6 and 
FHMIPv6 perform better compared to MIPv6 and FMIPv6 in term of Packet Delivery Ratio (PDR). For HMIPv6 and FHMIPv6, both have the same amount for the packet delivery ratio for various packet sizes. MIPv6 performs the least among these 4 mobility management protocols. The reason is because HMIPv6 and FHMIPv6 perform the MAP mechanism where the same hierarchical network does not need to be sent over to the higher hierarchical network. Whereas in FMIPv6 and FHMIPv6, the fast handover mechanism informs the new network about the handover process before it performs the handover processes. Thus, HMIPv6, FMIPv6 and FHMIPv6 perform better than the original MIPv6.

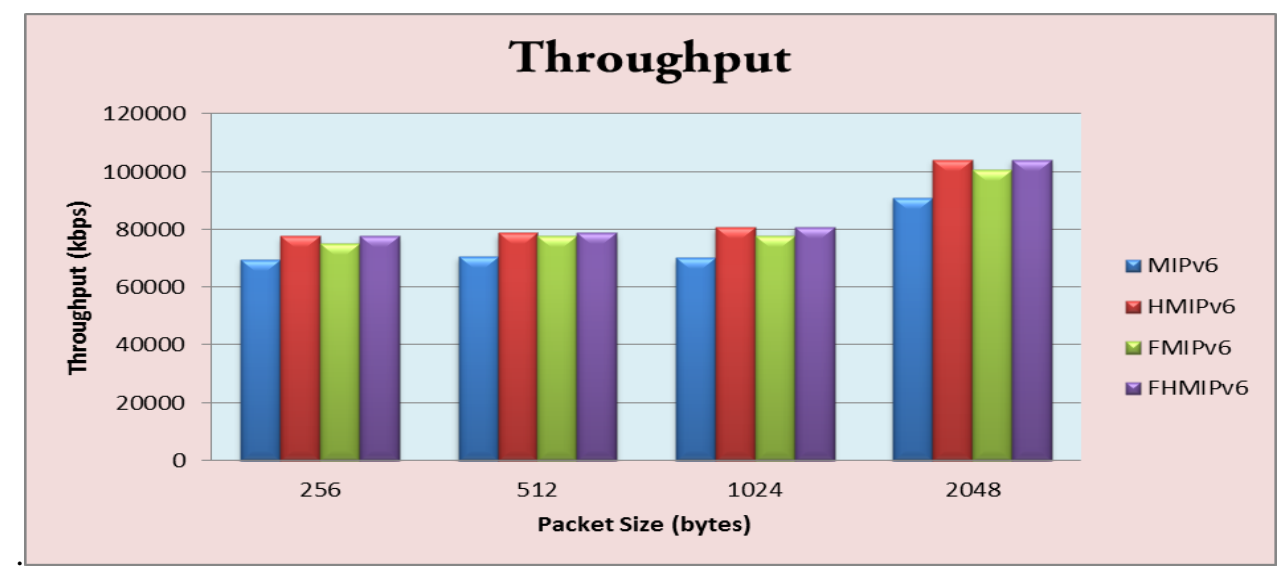

Fig. 4. Throughput Chart

Based on Figure 4, the throughput for all Host-Based mobility management protocols has been observed. The HMIPv6 and FHMIPv6 perform better compared to other two mechanisms. The HMIPv6 and FHMIPv6 share the same throughput for various packet sizes. For example packet size of 2048 bytes, HMIPv6 and FHMIPv6 have the throughput of $104133.43 \mathrm{Kbps}$. FMIPv6 has the throughput of $100761.60 \mathrm{Kbps}$ and MIPv6 has the throughput of 91023.06 Kbps. The reason of HMIPv6 and FHMIPv6 performs better than FMIPv6 and MIPv6 is explained as before where HMIPv6 and FHMIPv6 do not need to perform higher hierarchical data transmission if the nodes perform lower hierarchical network communication.

\section{Conclusion}

As conclusion, due to the various performance metrics results, the FHMIPv6 performs much better compares to others Host-Based mobility management protocols. By introducing this proposed expansion, it's able to improve service quality and service range of wireless communication in areas that are affected by coverage problems. In future, the Network-Based mobility management protocols such as Proxy Mobile Internet Protocol version 6 (PMIPv6) and Fast Proxy Mobile Internet Protocol version 6 (FPMIPv6) are proposed to be consolidate and make comparisons with Host-Based mobility management protocols in Wireless Mesh Network (WMN) environment. 


\section{References}

[1] D. Johnson, C. Perkins, and J. Arkko, "Mobility Support in IPv6," Internet Soc., Reston, VA, IETF RFC 3775, Jun. 2004.

[2] R. Koodli, "Fast Handovers for Mobile IPv6," Internet Soc., Reston, VA, IETF RFC 4068, Jul. 2005.

[3] H. Soliman, C. Castelluccia, K. ElMalki, and L. Bellier, "Hierarchical Mobile IPv6 Mobility Management (HMIPv6)," Internet Soc., Reston, VA, IETF RFC 4140, Aug. 2005.

[4] S. Hui and K. Yeung, "Challenges in the migration to $4 \mathrm{G}$ mobile systems," Commun. Mag. IEEE, December, pp. 54-59, 2003.

[5] Z. Chitedze and W. Tucker, "FHMIPv6-based handover for wireless mesh networks," in Proc. South. Africa Telecommun. Networks Appl. Conf., 2012, pp. 1-5.

[6] Jong-Hyouk Lee, Jean-Marie Bonnin, Ilsun You, and Tai-Myoung Chung, "Comparative Handover Performance Analysis of IPv6 Mobility Management Protocols," IEEE Transactions on Industrial Electronics, Vol. 60, No. 3, March 2013

[7] K Vasu, Sudipta Mahapatra, and C S Kumar, "MIPv6 Protocols: A Survey and Comparative Analysis", Computer Science \& Information Technology (CS \& IT) 07, pp. 73-93, 2012. (C) CS \& IT-CSCP 2012.

[8] J. H Sun, D. Howie and J. Sauvola, "Mobility Management Techniques for Next Generation Wireless Networks", Proc. of SPIE. Wireless and Mobile Communications, vol. 4586, pp. 155-166, 2012

[9] Zhang, Y. and H. Bi, 2012. "The Simulation of Hierarchical Mobile IPv6 with Fast Handover using NS2," Procedia Engineering, 37: 214-217.

[10]Gelogo, Y.E. and B. Park, "Reducing packet loss for mobile IPv6 fast handover (FMIPv6)," International Journal of Software Engineering and its Applications, 6(1): 8792, 2012.

[11] Ahmed, E., M. Shiraz and A. Gani, 2013. "Spectrum-aware Distributed Channel Assignment for Cognitive Radio Wireless Mesh Networks," Malaysian Journal of Computer Science, 26(3): 232-250.

[12] Byungjoo Park, Dongcheul Lee and Jaejin Lee, “AROSP: Advanced Route Optimization Scheme in PMIPv6 Networks for Seamless Multimedia Service", IJCSNS International Journal of Computer Science and Network Security, VOl.8, No.9, September 2008

[13] Muthut, S., B. Ong, N. Adilah, H. Zahri and R.B. Ahmad, 2015. "An Overview of Performance Enhancement of FHMIPv6 on Wireless Mesh Network," International Journal of Future Computer and Communication, 4(3): 160-164.

[14] Sashikumar Muthut, Bi-Lynn Ong, Wei Siang Hoh and R. Badlishah Ahmad,2015. “ Integration of Fast Handover and Hierarchical Mobile Internet Protocol with Wireless Mesh Network," Australian Journal of Basic and Applied Sciences, 9(25) Special 2015, Pages: $72-78$

[15] Wei Siang Hoh, Sashikumar Muthut, Bi-Lynn Ong, Mohamed Elshaikh, Mohd Nazri Mohd Warip and R. Badlishah Ahmad, 2015. "A Survey of Mobility Management Protocols," ARPN Journal of Engineering and Applied Sciencies, Vol.10, NO.19, October 2015. 